OPEN ACCESS

Edited by: Michael Sears,

Clemson University, United States

Reviewed by:

Mallory A. Ballinger,

University of California, Berkeley,

United States

Katharina Wollenberg Valero, University of Hull, United Kingdom

${ }^{*}$ Correspondence: Hiroshi Akashi

hiroshi-akashi@hotmail.co.jp

Specialty section

This article was submitted to Behavioral and Evolutionary Ecology,

a section of the journal

Frontiers in Ecology and Evolution

Received: 15 July 2020

Accepted: 14 May 2021

Published: 10 June 2021

Citation:

Akashi H (2021) Thermal Sensitivity of Heat Sensor TRPA1

Correlates With Temperatures Inducing Heat Avoidance Behavior

in Terrestrial Ectotherms.

Front. Ecol. Evol. 9:583837.

doi: 10.3389/fevo.2021.583837

\section{Thermal Sensitivity of Heat Sensor TRPA1 Correlates With Temperatures Inducing Heat Avoidance Behavior in Terrestrial Ectotherms}

\section{Hiroshi Akashi* \\ Department of Biological Science and Technology, Faculty of Industrial Science and Technology, Tokyo University of Science, Tokyo, Japan}

Temperature is an essential environmental factor that controls an organism's performances. As ectothermic animals largely rely on external heat sources for adjusting their body temperature, thermal perception is a primary process of behavioral thermoregulation. Transient receptor potential ankyrin 1 (TRPA1) is a heat sensitive ion channel in most non-mammalian species, and its heat activation has been suggested to induce heat avoidance behaviors in ectothermic animals. However, associations between TRPA1 and ecologically relevant temperatures have not been investigated, and the analyses including diverse taxa will provide robust support for understanding the associations. Here, I conducted extensive literature review, and assembled published data on thermal threshold of TRPA1 and three physiological parameters: the experimental voluntary maximum (EVM), which is body temperatures when heat avoidance behaviors are induced; the critical thermal maximum $\left(C T_{\max }\right)$, which is a point in temperature beyond which an organism becomes incapacitated; and average body temperature ( $T_{\text {mean }}$ ) recorded in the field. Then, I examined the relationships between thermal threshold of TRPA1 and each of the three physiological parameters. As phylogenetically closely related species tend to show similar trait values among species, I conducted the regression analyses by accounting for phylogenetic distances among species. This study supports previous research by affirming that thermal threshold of TRPA1 is substantially correlated with body temperature that the animals escaped from the heat source, represented here as EVM. Nevertheless, thermal threshold of TRPA1 showed a statistically insignificant correlation with $C T_{\text {max }}$ and $T_{\text {mean }}$. The results suggest that although thermal threshold of TRPA1 is evolutionarily labile, its associations with EVM is highly conserved among diverse terrestrial ectotherms. Therefore, thermal threshold of TRPA1 could be a useful parameter to evaluate species vulnerability to thermal stress particularly in the recent climate warming scenario.

Keywords: thermal biology, terrestrial ectotherms, thermoregulation, thermal sensitivity, transient receptor potential ankyrin 1, heat avoidance behavior, phylogenetic generalized least squares 


\section{INTRODUCTION}

Temperature is one of the most influential environmental factors that affects the organisms' performances. As ectothermic animals largely rely on external heat sources for adjusting their body temperature, thermal perception is an essential process of behavioral thermoregulation (Tattersall et al., 2012). Animals perceive temperature through sensory neurons in the peripheral nervous system, in which a subset of temperature-sensitive ion channels is expressed (Tominaga and Caterina, 2004; Dhaka et al., 2006). The transient receptor potential (TRP) ion channel superfamily consists of $\mathrm{Ca}^{2+}$ permeable non-selective cation channels, which function as polymodal receptors detecting mechanical, chemical, and thermal stimuli (Saito and Tominaga, 2017; Hoffstaetter et al., 2018). Among the TRP superfamily, a subset of TRPs (called thermoTRPs) is temperature sensitive, and known to participate in thermal perception in animals (Patapoutian et al., 2003; Venkatachalam and Montell, 2007). Recent studies reported that the activation temperatures of each thermoTRP are diverse among species, suggesting that the functions of thermoTRPs evolve with change in thermal physiology (Gracheva and Bagriantsev, 2015).

In electrophysiological analysis of the heterologous expression of thermoTRPs, thermal thresholds could be estimated from the Arrhenius plot that represents the logarithmic rate of inward currents against inverse temperatures in Kelvin (Voets et al., 2005). The intersection of lines fitted to baseline and linearly increasing currents of the Arrhenius plot indicates the initial temperature which activates thermoTRPs and it represents the thermal threshold of thermoTRPs (Boukalova et al., 2010; Gracheva et al., 2010; Vriens et al., 2014). The use of thermal thresholds for describing and comparing thermoTRPs has been discouraged because thermoTRPs are activated gradually rather than at a specific threshold and also vary unless the experimental conditions are kept constant across studies (Voets et al., 2005; Vriens et al., 2014). Although thermal thresholds of thermoTRPs should still be carefully discussed, they have been one of indices for describing ranges of thermal sensitivity (Gracheva and Bagriantsev, 2015). Studies on thermoTRPs have, in fact, revealed that each thermoTRP possesses channelspecific activation temperatures covering temperature ranges that animals normally experience (Patapoutian et al., 2003; Dhaka et al., 2006).

TRP ankyrin 1 (TRPA1) is a member of thermoTRPs and is among those which have been studied in a high number of species. Its thermal sensitivity has been debated in mammalian TRPA1, as cold activates TRPA1 in rodent species but not in primate species (Caspani and Heppenstall, 2009; Chen et al., 2013). In contrast, heat consistently activates TRPA1 in non-mammalian species except Caenorhabditis elegans (Chatzigeorgiou et al., 2010; Saito et al., 2014). In Drosophila melanogaster, four TRPA1 subtypes have been reported, and mutant analyses in larval or adult flies have demonstrated that the ortholog of vertebrate TRPA1 (dTRPA1) and Painless are requisite for avoiding noxious temperatures (Rosenzweig et al., 2005; Hamada et al., 2008; Neely et al., 2011; Peng et al., 2015). Recently, TRPA1 has been analyzed in conjunction with heat avoidance behaviors across various taxa, such as mosquitoes, Xenopus frogs, and Anolis lizards (Saito et al., 2016; Akashi et al., 2018; Li et al., 2019). These studies revealed a trend that species with higher thermal thresholds of TRPA1 showed heat avoidance behaviors induced at higher temperatures. These mutant and comparative analyses suggested that animals perceive noxious heat through the activation of TRPA1, which induces heat avoidance behavior.

Temperature-dependent performance of ectothermic animals is often graphically described using thermal reaction norms or thermal performance curves. Animals achieve their highest performance at the peak of the curve but become incapable of keeping right posture and performing at both extreme ends of the curve, which represent critical thermal maximum and minimum $\left(\mathrm{CT}_{\max }\right.$ and $\mathrm{CT}_{\min }$ ) (Cowles and Bogert, 1944; Huey and Stevenson, 1979; Lutterschmidt and Hutchison, 1997). The curve is typically asymmetric and left-skewed so that a deviation of body temperature from the optimum will reduce the performance greater toward the higher thermal limit than the lower thermal limit of the curve. Therefore, detection of temperature above the optimum is crucial. Animals accept the temperature rise or fall until their body temperature reaches the limit that they voluntarily tolerate. The highest and lowest voluntarily tolerable temperatures are defined as maximum and minimum voluntary tolerances, respectively, and the activity of animals takes place within thermal-safety margins bounded by the two voluntary tolerance temperatures (Cowles and Bogert, 1944; Vitt and Caldwell, 2008). The highest voluntary tolerance temperature is experimentally measured as experimental voluntary maximum (EVM) at which animals show the first attempt to escape from the heat sources (Ruibal, 1961; Hertz, 1981). $\mathrm{T}_{\text {mean }}$ is the mean body temperature recorded in the field and is not necessarily the temperature that maximizes performance (i.e., the peak of the thermal performance curve) as ectothermic animals regulate their body temperatures presumably through a compromise between costs and benefits associated with thermoregulatory behaviors (Huey and Slatkin, 1976; Huey and Stevenson, 1979).

Ectothermic animals, particularly thermoregulators, sense ambient temperatures to keep $\mathrm{T}_{\text {mean }}$ within thermal-safety margins distant from $\mathrm{CT}_{\max }$ and $\mathrm{CT}_{\min }$. TRPA1 is an excellent candidate molecule for the detection of temperature. Previous comparative studies have focused on the relationship between thermal threshold of TRPA1 and temperature that induces thermal avoidance behaviors (Saito et al., 2016; Akashi et al., 2018; Li et al., 2019). However, these studies have not considered whether thermal threshold of TRPA1 is associated with other ecologically relevant parameters, such as $\mathrm{CT}_{\max }$ and $\mathrm{T}_{\text {mean }}$. In addition, ecological or physiological similarities among species would arise either from adaptation to similar environments or from close phylogenetic relationships (Cavender-Bares et al., 2004; Losos, 2008; Cooper et al., 2011; Kellermann et al., 2012), thus, comparative analyses should consider the phylogenetic distances among species (Felsenstein, 1985; Hansen and Orzack, 2005). The previous studies might have overlooked the possibility of correlations between the physiological properties of TRPA1 and other physiological parameters. 
In this study, I assembled published data regarding thermal threshold of TRPA1 and the physiological traits EVM, $\mathrm{CT}_{\max }$, and $\mathrm{T}_{\text {mean }}$, in ectothermic animals. Then, I analyzed whether thermal threshold of TRPA1 correlates with any of the relevant physiological traits by conducting ordinary least squares (OLS) and phylogenetic generalized least squares (PGLS) analyses to control the effects of phylogenetic distances. Here, I found that thermal threshold of TRPA1 correlates specifically with EVM even after considering phylogenetic distances among species, but not with $\mathrm{CT}_{\max }$ nor $\mathrm{T}_{\text {mean }}$. Therefore, the current study suggests that the association between TRPA1 and EVM is highly conserved among diverse terrestrial ectotherms, and TRPAl contributes in part to setting the upper limit of species thermal-safety margins.

\section{MATERIALS AND METHODS}

\section{Literature Review and Data Acquisition}

I searched National Center for Biotechnology Information $(\mathrm{NCBI})^{1}$ for studies reported complete coding sequences of and physiological properties of TRPA1, and also Google Scholar ${ }^{2}$ and PubMed ${ }^{3}$ for studies on TRPA1 which included its thermal threshold as an index of thermal sensitivity. The literature was searched for studies published before February 28, 2021. The query term for NCBI was "TRPA1." The query terms for Google Scholar and PubMed were the following: trp, "temperature threshold" OR "thermal threshold," and "Arrhenius plot." The technical terms were double quoted. Two phrases were linked with "OR" and queried if the phrases were equally used across studies. For species whose thermal thresholds of TRPA1 have been reported, I conducted further literature surveys with the following terms to identify studies reporting (1) EVM, (2) $\mathrm{CT}_{\max }$, and (3) $\mathrm{T}_{\text {mean }}$. The query terms were the following: (1) "species," "voluntary maximum" OR "maximum voluntary" OR nocifensive response behavior; (2) "species," "critical maximum" OR "critical thermal maximum" OR $\mathrm{CT}_{\max }$; (3) "species" field average body temperature. Plural forms were also queried. Ectothermic species were included for subsequent analyses if thermal threshold of TRPA1 and/or at least one of the three physiological parameters had been reported.

I summarized the reported values by calculating a single grand mean and grand standard error (SE) for each trait and species. This calculation requires means, errors (SE or standard deviations, SD), and sample sizes to be reported in the original papers. However, several studies presented the data in figures rather than as numerical values. Thus, depending on the reported data set, I defined the grand mean and grand SE as follows: (a) For data reported by multiple and comprehensive studies, I summarized the data by calculating the grand means and grand SE using total data sets within each trait for each species. The data with missing SE or SD and sample size were omitted from the grand means and grand SE. (b) For data reported by multiple but incomprehensive studies (i.e., lack of SE or SD and sample size

\footnotetext{
${ }^{1}$ https://www.ncbi.nlm.nih.gov/

${ }^{2}$ https://scholar.google.com/

${ }^{3}$ https://pubmed.ncbi.nlm.nih.gov/
}

in all the reported studies), I calculated the mean and SE of the reported means as the grand mean and grand SE, respectively. (c) For data reported by a single study, the mean and SE were treated as the grand mean and grand SE, respectively. If the data lacked SE or SD and sample size was not indicated, their mean was used as the grand mean without an error.

As thermal threshold of TRPA1 was reported from two different electrophysiological approaches using HEK293 cells or Xenopus oocytes, the data obtained from HEK293 cells and Xenopus oocytes and those obtained only from Xenopus oocytes were separately summarized. If different isoforms were investigated in the electrophysiological analyses, the data for the longer isoform was adopted for the current study. All four parameters reviewed here should not be taken as fixed values for the species as each value varied across or within studies to some extent, depending on experimental protocols. All statistical analyses were performed using the $\mathrm{R}$, version 3.5.1 ( $\mathrm{R}$ Development Core Team, 2018).

\section{Phylogenetic Analysis}

Coding sequences of TRPA 1 for 18 species that passed the criteria of the literature review were obtained from GenBank ${ }^{4}$. The accession numbers of these sequences are shown in Figure $\mathbf{1 .}$ I translated the nucleotide sequences to amino acid sequences and constructed the multiple protein sequence alignments with MUSCLE (Edgar, 2004), using default penalties for a gap-opening $(-2.9)$, a gap-extension (0), and hydrophobicity multiplier (1.2). For phylogenetic analysis, the aligned amino acid sequences were converted back to nucleotide sequences. Then, I inferred a phylogenetic tree from the aligned nucleotide sequences by using the maximum likelihood method with 1,000 bootstrap replicates in MEGA X (Kumar et al., 2018; Stecher et al., 2020). The best nucleotide substitution model for maximum likelihood method was determined using the "find best DNA/protein models" tool, and the Kimura 2-parameter model of sequence evolution under a gamma distribution of rates with invariant sites was adopted based on the lowest Bayesian information criterion scores yielded from the analysis (Kimura, 1980). All positions with less than $95 \%$ site coverage were eliminated, resulting in a total of 3,177 positions in the final dataset for the analysis. TRPA1 of Patiria pectinifera was used as the outgroup.

\section{Evaluating Phylogenetic Signal and Correlations Between Physiological Parameters}

I assessed whether phylogenetic distances explain the similarity and divergence in thermal threshold of TRPA1 and the physiological parameters (EVM, $\mathrm{CT}_{\max }$, and $\mathrm{T}_{\text {mean }}$ ) by means of Pagel's lambda $(\lambda)$ (Pagel, 1999). Lambda scales the internal branches relative to the tip branches of the phylogenetic tree from 1 to 0 , inferring the extent of the phylogenetic signal. Lambda equal to 1 indicates that traits evolve under a Brownian motion model and closely related species are expected to have similar trait values. Lambda equal to 0 indicates that traits

\footnotetext{
${ }^{4}$ http://www.ncbi.nlm.nih.gov/genbank/
} 


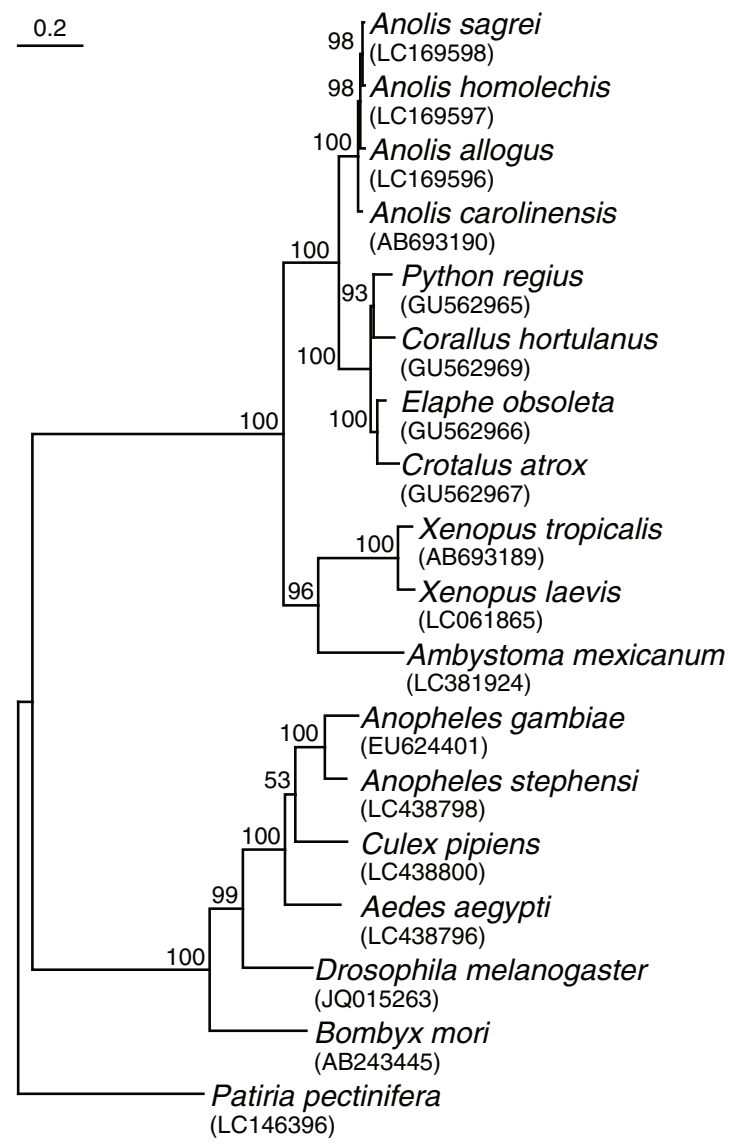

FIGURE 1 | Inferred phylogenetic tree of 18 species reviewed in the analyses. The tree was estimated from the maximum likelihood $(\mathrm{ML})$ analysis of TRPA1 coding sequences. The numbers at each node indicate bootstrap values obtained from 1,000 replicates using the ML method. Scale bar refers to a phylogenetic distance of 0.2 nucleotide substitutions per site. Accession numbers are shown in parentheses.

evolve independently of the phylogeny and signifies the absence of phylogenetic signal. In this analysis, the inferred maximum likelihood tree was first imported into the $\mathrm{R}$ by using the $\mathrm{R}$ package "ape" (Paradis et al., 2004). Lambda was then calculated from the grand mean and grand SE for each trait by using the phylosig function in the R package "phytools" (Revell, 2012). For thermal threshold of TRPA1, lambda was calculated using the species with inferred phylogeny. The studies reporting thermal threshold of TRPA1 did not have a complete set of physiological parameters (i.e., EVM, $\mathrm{CT}_{\max }$, and $\mathrm{T}_{\text {mean }}$ ). Species without those parameters were pruned from the inferred phylogeny, and lambda was calculated for $\mathrm{EVM}, \mathrm{CT}_{\max }$, and $\mathrm{T}_{\text {mean }}$ using the remaining species with the corresponding pruned phylogeny.

I investigated the relationship between the grand means of thermal threshold of TRPA1 and the physiological parameters $\left(\mathrm{CT}_{\max }, \mathrm{EVM}\right.$, and $\left.\mathrm{T}_{\text {mean }}\right)$ by conducting OLS and PGLS regression analyses. As the activation of TRPA1 was assumed to regulate the physiological parameters, thermal threshold of TRPA1 was selected as the sole explanatory variable, and the three physiological parameters were analyzed as response variables. I first fitted the OLS regression to the data by using the $\operatorname{lm}$ function in $\mathrm{R}$ so that the fitted regression line indicated how well an explanatory variable was associated with the response variables. Residual error in OLS regression model needs to be independent among observations. However, the error will no longer be independent particularly in the case of interspecific comparisons. PGLS analysis considers the phylogenetic relationship based on an assumption that the residual error in the regression model is proportional to the internal branch length of the phylogenetic tree (Felsenstein, 1985; Revell, 2010). Thus, I secondly conducted three independent PGLS analyses on thermal threshold of TRPA1 paired with one of the three parameters and each of the corresponding pruned phylogenies by using the pgls function in the R package "caper" (Orme et al., 2018). The PGLS analysis estimates the maximum likelihood values of lambda in the residual error simultaneously with the regression parameters.

\section{RESULTS}

The literature review showed that thermal threshold of TRPA1 has been estimated from the Arrhenius plot in 18 ectothermic species (Supplementary Table 1). Among those species, the physiological parameters (EVM, $\mathrm{CT}_{\max }$, and $\mathrm{T}_{\text {mean }}$ ) were reported for 9, 7, and 6 species, respectively (Supplementary Table 2). The grand mean and grand SE from both HEK293 cells and Xenopus oocytes and those only from Xenopus oocytes are summarized in Table $\mathbf{1}$ and Supplementary Table 3, respectively. In the former case, Pagel's lambda for thermal threshold of TRPA1 was 0.786 , and the null hypothesis of no phylogenetic signal (i.e., $\lambda=0)$ was rejected $(P=0.010)$. EVM also showed a high value of lambda $(\lambda=0.826)$ but with insignificant $P$-value $(P=0.192)$. Both $\mathrm{CT}_{\max }$ and $\mathrm{T}_{\text {mean }}$ showed lambda to be 0 with $P$-value of 1.0. In addition to estimating lambda for each trait, OLS and PGLS analyses were conducted for each of the three combinations of the two parameters (Figure 2 and Table 2). OLS analysis showed a significant positive correlation between thermal threshold of TRPA1 and EVM (slope $=0.603, P=0.014$ ) (Figure 2A). However, thermal threshold of TRPA1 was not correlated with $\mathrm{CT}_{\max }$ nor $\mathrm{T}_{\text {mean }}$ $\left(\mathrm{CT}_{\max }:\right.$ slope $=-0.464, P=0.187 ; \mathrm{T}_{\text {mean }}:$ slope $=0.166$, $P=0.524$ ) (Figures 2B,C). Although PGLS analysis detected strong phylogenetic signal in the model of thermal threshold of TRPA1 and EVM $\left(\mathrm{ML}_{\mathrm{L}} \lambda=0.904\right)$, thermal threshold of TRPA1 was positively correlated with EVM as observed in OLS analysis (slope $=0.458, P=0.026$ ) (Figure 2A). PGLS analysis did not show a significant correlation between thermal threshold of TRPA1 and $\mathrm{CT}_{\max }$ nor $\mathrm{T}_{\text {mean }}\left(\mathrm{CT}_{\max }\right.$ : slope $=-0.461, P=0.184$; $\mathrm{T}_{\text {mean }}$ : slope $=0.212, P=0.511$ ) with no phylogenetic signal in these traits (Figures 2B,C). The OLS and PGLS analyses conducted on data obtained only from Xenopus oocytes showed the same trend described above (Supplementary Table 4).

\section{DISCUSSION}

Here, I investigated the relationship between thermal threshold of TRPA1 and the physiological traits (EVM, $\mathrm{CT}_{\max }$, and $\mathrm{T}_{\text {mean }}$ ) 
TABLE 1 | Summary of the grand mean \pm grand standard error for each trait used in the analyses.

\begin{tabular}{|c|c|c|c|c|c|}
\hline Species & TRPA1 & EVM & $\mathrm{CT}_{\max }$ & $\mathbf{T}_{\text {mean }}$ & References \\
\hline Crotalus atrox & $27.6 \pm 0.9(6)^{c}$ & $34(-)^{c}$ & $39(-)^{c}$ & $27.4(-)^{\mathrm{c}}$ & Brattstrom, 1965; Gracheva et al., 2010 \\
\hline Elaphe obsoleta & $37.9 \pm 0.4(18)^{a}$ & $38(-)^{\mathrm{c}}$ & $38(-)^{\mathrm{c}}$ & $27 \pm 0.4(158)^{a}$ & $\begin{array}{l}\text { Brattstrom, 1965; Blouin-Demers and Weatherhead, } \\
\text { 2001; Gracheva et al., 2010; Kurganov et al., } 2017\end{array}$ \\
\hline Python regius & $32.7 \pm 0.5(6)^{c}$ & - & - & - & Gracheva et al., 2010 \\
\hline Corallus hortulanus & $29.6 \pm 0.3(6)^{c}$ & - & - & - & Gracheva et al., 2010 \\
\hline Anolis carolinensis & $35.2 \pm 0.5(35)^{\mathrm{a}}$ & $36.0 \pm 0.7(15)^{a}$ & $41.7 \pm 0.2(49)^{a}$ & $29.8 \pm 0.3(39)^{a}$ & $\begin{array}{l}\text { Brattstrom, 1965; Corn, 1971; Wilson and Echternacht, } \\
\text { 1990; Lailvaux and Irschick, 2007; Saito et al., 2012; } \\
\text { Kurganov et al., } 2014\end{array}$ \\
\hline Anolis allogus & $33.5 \pm 0.7(26)^{c}$ & $32.3 \pm 0.2(47)^{a}$ & - & $28.3 \pm 0.1(248)^{\mathrm{a}}$ & Ruibal, 1961; Schettino et al., 2010; Akashi et al., 2018 \\
\hline Anolis homolechis & $36.4 \pm 0.8(18)^{c}$ & $34.5 \pm 0.3(53)^{\mathrm{a}}$ & - & $29.4 \pm 0.1(276)^{\mathrm{a}}$ & Ruibal, 1961; Schettino et al., 2010; Akashi et al., 2018 \\
\hline Anolis sagrei & $36.1 \pm 0.8(22)^{\mathrm{c}}$ & $34.1 \pm 0.3(9)^{\mathrm{c}}$ & $39.4 \pm 0.1(102)^{a}$ & $32.0 \pm 0.2(295)^{a}$ & $\begin{array}{l}\text { Ruibal, 1961; Corn, 1971; Schettino et al., 2010; } \\
\text { Akashi et al., 2018; Gunderson et al., } 2020\end{array}$ \\
\hline Xenopus tropicalis & $39.8 \pm 0.4(44)^{a}$ & $40(4)^{\mathrm{c}}$ & $34(18)^{\mathrm{c}}$ & - & $\begin{array}{l}\text { Herrel and Bonneaud, 2012; Saito et al., 2012; Saito } \\
\text { et al., } 2016\end{array}$ \\
\hline Xenopus laevis & $37.5 \pm 0.4(51)^{a}$ & $38(4)^{c}$ & $31.6 \pm 0.1(51)^{a}$ & - & $\begin{array}{l}\text { Sherman and Levitis, 2003; Saito et al., 2016; Araspin } \\
\text { et al., } 2020\end{array}$ \\
\hline Ambystoma mexicanum & $39.7 \pm 1.0(6)^{\mathrm{C}}$ & - & - & - & Oda et al., 2019 \\
\hline Drosophila melanogaster & $29.7 \pm 0.3(-)^{\mathrm{c}}$ & $30.2 \pm 0.6(-)^{b}$ & $41.0 \pm 0.1(12)^{a}$ & - & $\begin{array}{l}\text { Folk et al., 2007; Wang et al., 2008; Oswald et al., } \\
\text { 2011; Kang et al., } 2012\end{array}$ \\
\hline Bombyx mori & $21.6(-)^{c}$ & - & - & - & Sato et al., 2014 \\
\hline Anopheles gambiae & $28.5 \pm 0.7(38)^{\mathrm{c}}$ & - & - & - & Li et al., 2019 \\
\hline Anopheles stephensi & $30.3 \pm 0.9(17)^{\mathrm{c}}$ & - & - & - & Li et al., 2019 \\
\hline Aedes aegypti & $32.0 \pm 0.8(18)^{\mathrm{c}}$ & - & - & - & Li et al., 2019 \\
\hline Culex pipiens pallens & $21.8 \pm 0.7(20)^{\mathrm{C}}$ & - & - & - & Li et al., 2019 \\
\hline Patiria pectinifera & $34.8 \pm 0.5(23)^{c}$ & - & - & - & Saito et al., 2017 \\
\hline
\end{tabular}

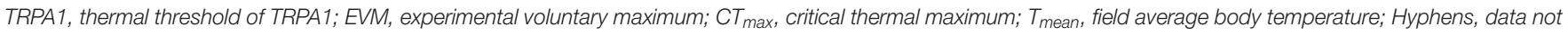
available. Letters in upper case denotes the criteria ( $a, b$, and c) used for the data summary as described in method.

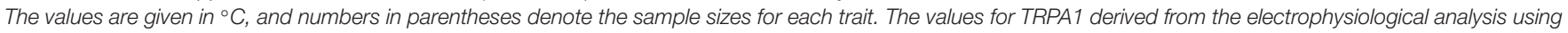
HEK293 cells and Xenopus oocytes.

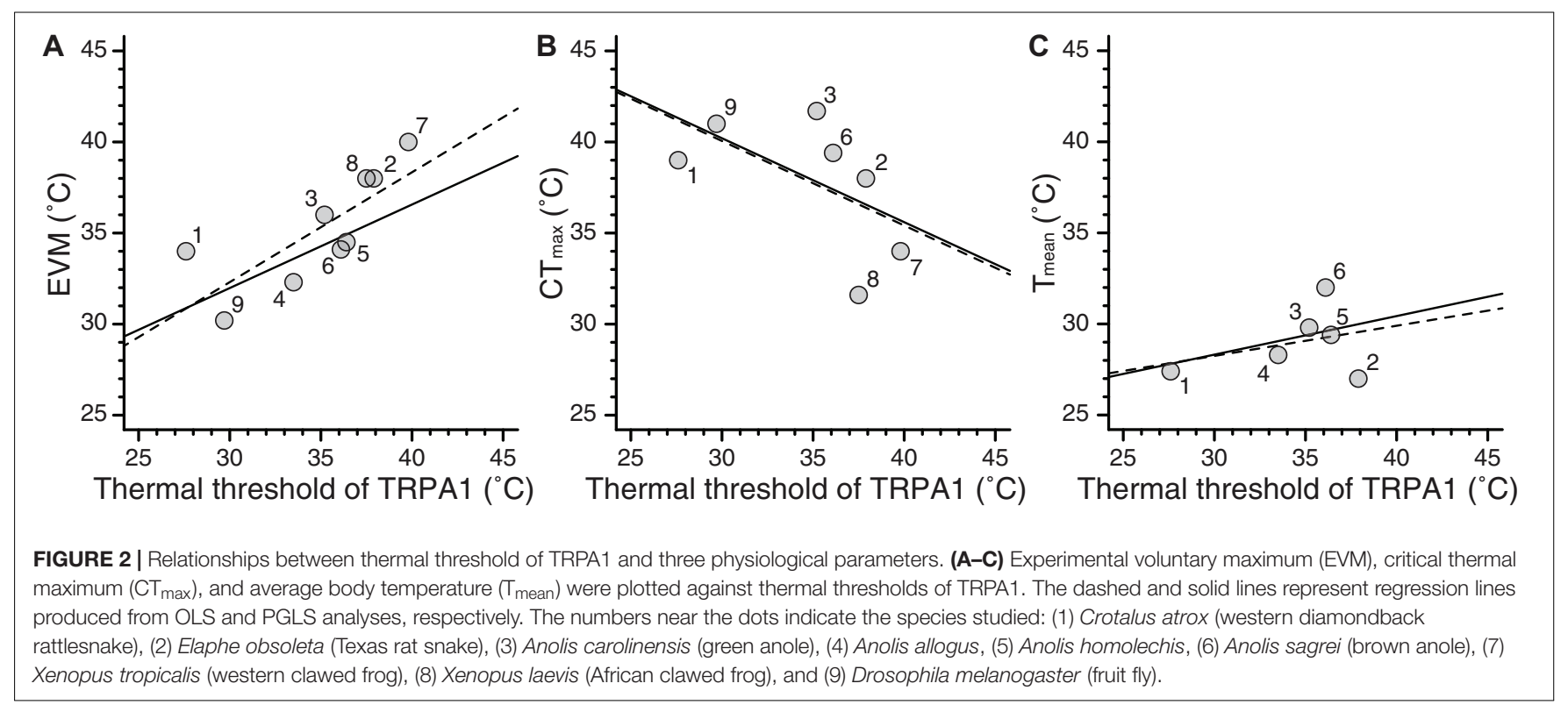

in terrestrial ectotherms by conducting OLS and PGLS analyses. Previous studies on individual species or sets of closely related species suggested that the activation of TRPA1 induces heat avoidance behaviors (Rosenzweig et al., 2005; Hamada et al.,
2008; Neely et al., 2011; Saito et al., 2016; Akashi et al., 2018; $\mathrm{Li}$ et al., 2019). The regression analyses also supported the previous findings even with the inclusion of diverse taxa, and revealed that thermal threshold of TRPA1 was specifically related 
TABLE 2 | Results of ordinary least squares (OLS) and phylogenetic generalized least squares (PGLS) analyses performed on the data obtained from HEK293 cells and Xenopus oocytes.

\begin{tabular}{|c|c|c|c|c|c|c|c|}
\hline \multirow[b]{2}{*}{ Model } & \multirow[b]{2}{*}{ Framework } & \multicolumn{2}{|c|}{ Intercept } & \multicolumn{2}{|c|}{ Slope } & \multirow[b]{2}{*}{$R^{2}$} & \multirow[b]{2}{*}{$M L \lambda$} \\
\hline & & Estimate \pm SE & $P$ & Estimate \pm SE & $P$ & & \\
\hline \multirow[t]{2}{*}{ EVM $\sim$ TRPA1 } & OLS & $14.21 \pm 6.48$ & 0.065 & $0.6 \pm 0.18$ & 0.014 & 0.6 & $\mathrm{n} / \mathrm{a}$ \\
\hline & PGLS & $18.23 \pm 5.88$ & 0.017 & $0.46 \pm 0.16$ & 0.026 & 0.53 & 0.904 \\
\hline \multirow[t]{2}{*}{$\mathrm{CT}_{\max } \sim \mathrm{TRPA} 1$} & OLS & $54.0 \pm 10.66$ & 0.004 & $-0.46 \pm 0.30$ & 0.187 & 0.32 & $\mathrm{n} / \mathrm{a}$ \\
\hline & PGLS & $54.0 \pm 10.42$ & 0.004 & $-0.46 \pm 0.30$ & 0.184 & 0.32 & 0 \\
\hline \multirow[t]{2}{*}{$\mathrm{T}_{\text {mean }} \sim \mathrm{TRPA} 1$} & OLS & $23.28 \pm 8.2$ & 0.047 & $0.17 \pm 0.24$ & 0.524 & 0.11 & $\mathrm{n} / \mathrm{a}$ \\
\hline & PGLS & $21.96 \pm 10.3$ & 0.1 & $0.21 \pm 0.29$ & 0.51 & 0.11 & 0 \\
\hline
\end{tabular}

Significant correlations are shown in boldface; n/a, not applicable.

to EVM rather than to $\mathrm{CT}_{\max }$ and $\mathrm{T}_{\text {mean }}$. The results suggest that although thermal threshold of TRPA1 is evolutionarily labile, its association with EVM is highly conserved among diverse terrestrial ectotherms. Animals stay active within thermal range bounded by maximum and minimum voluntarily tolerable temperatures (Vitt and Caldwell, 2008); thus, TRPA1 may be involved in defining the upper limit of species thermalsafety margins.

EVM is, by definition, lower than $\mathrm{CT}_{\max }$ as the animals should retreat from heat sources before they become incapable of keeping right posture due to the heat stress. Therefore, thermal threshold of TRPA1 may have been kept lower than $\mathrm{CT}_{\max }$ through adaptive evolution, allowing the animals to perceive stressful temperatures before experiencing lethal temperatures. The insignificant correlation between thermal threshold of TRPA1 and $\mathrm{CT}_{\max }$, otherwise, suggests that these two parameters evolve independent of one another, such that the other sensory molecules are responsible for perceiving the lethal temperatures. Field body temperature of ectothermic animals tends to fluctuate throughout a day, season, and also along a thermal environmental gradient and thus should ideally be assessed by a large number of observations, e.g., observations on at least 10 individuals in the case of lizards (Hertz et al., 2013). In the current study, each of the grand mean for $\mathrm{T}_{\text {mean }}$ derived from extensive field observations except for C. atrox (Table 1). Nevertheless, $\mathrm{T}_{\text {mean }}$ did not yield any significant correlation with thermal threshold of TRPA1. As ectothermic animals adjust their body temperatures depending on costs and benefits associated with thermoregulation, such as intra- and/or interspecific competition (Huey and Slatkin, 1976; Huey and Stevenson, 1979), the ectothermic animals may not be able to achieve or adjust their body temperatures, depending solely on temperature perceived through TRPA1.

While thermal threshold of TRPA1 and EVM were significantly correlated one another, that of TRPA1 in some species deviated from EVM in a few Celsius degrees. In other words, heat avoidance behaviors seem not to be induced exactly at thermal threshold of TRPA1. This could be true but alternate explanations are also possible. Thermal thresholds of TRPA1 assessed in the current study derived from the electrophysiological analysis conducted on heterologously expressed TRPA1 using Xenopus oocytes or HEK293 cells. Two-electrode voltage clamp or patch-clamp recordings are particularly useful for analyzing the physiological properties of ion channels although cell physiology, such as the ion compositions across cell membranes and/or the membrane potentials, varies depending upon the experimental protocols. The artificial cell physiology could result in the deviation of thermal thresholds of TRPA1 from those in vivo. Based on $\mathrm{Ca}^{2+}$ imaging experiments, for example, a TRPA1 agonist-responsive neurons in dorsal root ganglions (DRG) increased intracellular calcium concentration at a threshold a few degrees lower than thermal thresholds of TRPA1 estimated from heterologous expression systems in chickens (Saito et al., 2014). In addition, the involvement of the other molecules may account for an offset of few Celsius degrees between the two parameters. In DRG neurons, the other thermoTRP channel, TRP vanilloid 1 (TRPV1), is often co-expressed with TRPA1 and has also been suggested as a heat sensitive channel in native DRG neurons as well as TRPA1 (Story et al., 2003; Prober et al., 2008; Saito et al., 2012, 2014). In the case of fruit flies, four TRP channels belong to the TRPA subfamily, and Painless in addition to TRPA1 was also reported to be involved in avoidance of noxious heat (Neely et al., 2011). Thus, the integration of these heat-sensitive channels may better explain thermal sensitivities at whole-animal levels.

Although thermal thresholds of TRPA1 were analyzed in the current study, other physiological properties of thermoTRPs could also be important parameters. For instance, a member of thermoTRP channels, TRP melastatin 8 (TRPM8), showed that its half-maximal activation temperatures, rather than its thermal threshold, were correlated with core body temperatures in chicken, rat, and two Xenopus species, X. laevis and X. tropicalis (Myers et al., 2009). In addition, the thermal sensitivities of TRPA1, as characterized by normalized current sizes, were less sensitive to heat in the presumed hot-adapted species, $X$. tropicalis and $X$. borealis, compared with the presumed coldadapted species, X. laevis and X. muelleri (Saito et al., 2019). As such, other physiological properties of thermoTRPs may also provide keys to account for species thermal biology.

In this study, the regression analyses on diverse taxa showed that the values of EVM can be explained as a function of thermal threshold of TRPA1. Therefore, the study on thermal physiological properties of TRPA1 may help evaluate species' vulnerability to thermal stress that is not always easy to measure from behavioral experiments due to limitations of animal handling, such as substantially large or small body size, conditions under captivity, or the conservation status. 
In these cases, the thermal physiological properties of TRPA1 could be useful particularly in the recent climate warming scenario, since electrophysiological analysis can be conducted if the nucleotide sequences of target thermoTRPs have been reported. Further studies on thermoTRPs both from physiological and ecological perspectives are expected and will lead to a better understanding of thermoTRPs' functional evolution regarding species' thermal ecology.

\section{DATA AVAILABILITY STATEMENT}

The datasets presented in this study can be found in online repositories. The names of the repository/repositories and accession number(s) can be found in the article/ Supplementary Material.

\section{AUTHOR CONTRIBUTIONS}

The author confirms the sole responsibility for the following: study conception and design, data collection, analysis and interpretation of the results, and manuscript preparation.

\section{REFERENCES}

Akashi, H. D., Saito, S., Cádiz Díaz, A., Makino, T., Tominaga, M., and Kawata, M. (2018). Comparisons of behavioural and TRPA1 heat sensitivities in three sympatric Cuban Anolis lizards. Mol. Ecol. 27, 2234-2242. doi: 10.1111/mec. 14572

Araspin, L., Martinez, A. S., Wagener, C., Courant, J., Louppe, V., Padilla, P., et al. (2020). Rapid shifts in the temperature dependence of locomotor performance in an invasive frog, Xenopus laevis, implications for conservation. Integr. Comp. Biol. 60, 456-466. doi: 10.1093/icb/icaa010

Blouin-Demers, G., and Weatherhead, P. J. (2001). An experimental test of the link between foraging, habitat selection and thermoregulation in black rat snakes Elaphe obsoleta obsoleta. J. Anim. Ecol. 70, 1006-1013. doi: 10.1046/j.00218790.2001.00554.x

Boukalova, S., Marsakova, L., Teisinger, J., and Vlachova, V. (2010). Conserved residues within the putative S4-S5 region serve distinct functions among thermosensitive vanilloid transient receptor potential (TRPV) channels. J. Biol. Chem. 285, 41455-41462. doi: 10.1074/jbc.m110.145466

Brattstrom, B. H. (1965). Body temperatures of reptiles. Am. Midl. Nat. 73, 376-422. doi: $10.2307 / 2423461$

Caspani, O., and Heppenstall, P. A. (2009). TRPA1 and cold transduction: an unresolved issue? J. Gen. Physiol. 133, 245-249. doi: 10.1085/jgp.200810136

Cavender-Bares, J., Ackerly, D. D., Baum, D. A., and Bazzaz, F. A. (2004). Phylogenetic overdispersion in Floridian oak communitites. Am. Nat. 163, 823-843. doi: $10.1086 / 386375$

Chatzigeorgiou, M., Yoo, S., Watson, J. D., Lee, W. H., Spencer, W. C., Kindt, K. S., et al. (2010). Specific roles for DEG/ENaC and TRP channels in touch and thermosensation in C. elegans nociceptors. Nat. Neurosci. 13, 861-868. doi: $10.1038 / \mathrm{nn} .2581$

Chen, J., Kang, D., Xu, J., Lake, M., Hogan, J. O., Sun, C., et al. (2013). Species differences and molecular determinant of TRPA1 cold sensitivity. Nat. Commun. 4:2501. doi: 10.1038/ncomms3501

Cooper, N., Freckleton, R. P., and Jetz, W. (2011). Phylogenetic conservatism of environmental niches in mammals. Proc. R. Soc. B. 278, 2384-2391. doi: 10.1098/rspb.2010.2207

Corn, M. J. (1971). Upper thermal limits and thermal preferenda for three sympatric species of Anolis. J. Herpetol. 5, 17-21. doi: 10.2307/1562838

\section{FUNDING}

This work was supported by Grant-in-Aid for JSPS Fellows (Grant Number JP19J01186), Fostering Joint International Research B (Grant Number JP19KK0184), and Grant-in-Aid for Early-Career Scientists (Grant Number JP21K15166) from Japan Society for the Promotion of Science (JSPS).

\section{ACKNOWLEDGMENTS}

I warmly thank Dr. Shinichi Miyagawa and his lab members for helpful comments for the current study. I also thank the editor and reviewers for their helpful feedback, which greatly improved this manuscript.

\section{SUPPLEMENTARY MATERIAL}

The Supplementary Material for this article can be found online at: https://www.frontiersin.org/articles/10.3389/fevo.2021. 583837/full\#supplementary-material

Cowles, R. B., and Bogert, C. M. (1944). A preliminary study of the thermal requirements of desert reptiles. Bull. Am. Museum Nat. Hist. 83, 261-296.

Dhaka, A., Viswanath, V., and Patapoutian, A. (2006). TRP ion channels and temperature sensation. Annu. Rev. Neurosci. 29, 135-161. doi: 10.1146/ annurev.neuro.29.051605.112958

Edgar, R. C. (2004). MUSCLE: multiple sequence alignment with high accuracy and high throughput. Nucleic Acids Res. 32, 1792-1797. doi: 10.1093/nar/ gkh340

Felsenstein, J. (1985). Phylogenies and the comparative method. Am. Nat. 125, 1-15. doi: 10.1086/284325

Folk, D. G., Hoekstra, L. A., and Gilchrist, G. W. (2007). Critical thermal maxima in knockdown-selected Drosophila: are thermal endpoints correlated? J. Exp. Biol. 210, 2649-2656. doi: 10.1242/jeb.003350

Gracheva, E. O., and Bagriantsev, S. N. (2015). Evolutionary adaptation to thermosensation. Curr. Opin. Neurobiol. 34, 67-73. doi: 10.1016/j.conb.2015. 01.021

Gracheva, E. O., Ingolia, N. T., Kelly, Y. M., Cordero-Morales, J. F., Hollopeter, G., Chesler, A. T., et al. (2010). Molecular basis of infrared detection by snakes. Nature 464, 1006-1011. doi: 10.1038/nature08943

Gunderson, A. R., Fargevieille, A., and Warner, D. A. (2020). Egg incubation temperature does not influence adult heat tolerance in the lizard Anolis sagrei. Biol. Lett. 16:20190716. doi: 10.1098/rsbl.2019.0716

Hamada, F. N., Rosenzweig, M., Kang, K., Pulver, S. R., Ghezzi, A., Jegla, T. J., et al. (2008). An internal thermal sensor controlling temperature preference in Drosophila. Nature 454, 217-220. doi: 10.1038/nature07001

Hansen, T. F., and Orzack, S. H. (2005). Assessing current adaptation and phylogenetic inertia as explanations of trait evolution: the need for controlled comparisons. Evolution 59, 2063-2072. doi: 10.1554/05-088.1

Herrel, A., and Bonneaud, C. (2012). Temperature dependence of locomotor performance in the tropical clawed frog, Xenopus tropicalis. J. Exp. Biol. 215, 2465-2470. doi: 10.1242/jeb.069765

Hertz, P. E. (1981). Adaptation to altitude in two West Indian anoles (Reptilia: Iguanidae): field thermal biology and physiological ecology. J. Zool. Lond. 195, 25-37. doi: 10.1111/j.1469-7998.1981.tb01891.x

Hertz, P. E., Arima, Y., Harrison, A., Huey, R. B., Losos, J. B., and Glor, R. E. (2013). Asynchronous evolution of physiology and morphology in Anolis lizards. Evolution 67, 2101-2113. doi: 10.1111/evo.12072 
Hoffstaetter, L. J., Bagriantsev, S. N., and Gracheva, E. O. (2018). TRPs et al.: a molecular toolkit for thermosensory adaptations. Pflugers Arch. Eur. J. Physiol. 470, 745-759. doi: 10.1007/s00424-018-2120-5

Huey, R. B., and Slatkin, M. (1976). Cost and benefits of lizard thermoregulation. Q. Rev. Biol. 51, 363-384. doi: 10.1086/409470

Huey, R. B., and Stevenson, R. D. (1979). Integrating thermal physiology and ecology of ectotherms: a discussion of approaches. Amer. Zool. 19, 357-366. doi: 10.1093/icb/19.1.357

Kang, K., Panzano, V. C., Chang, E. C., Ni, L., Dainis, A. M., Jenkins, A. M., et al. (2012). Modulation of TRPA1 thermal sensitivity enables sensory discrimination in Drosophila. Nature 481, 76-80. doi: 10.1038/nature10715

Kellermann, V., Overgaard, J., Hoffmann, A. A., Fljøgaard, C., Svenning, J. C., and Loeschcke, V. (2012). Upper thermal limits of Drosophila are linked to species distributions and strongly constrained phylogenetically. Proc. Natl. Acad. Sci. U. S. A. 109, 16228-16233. doi: 10.1073/pnas.1207553109

Kimura, M. (1980). A simple method for estimating evolutionary rates of base substitutions through comparative studies of nucleotide sequences. J. Mol. Evol. 16, 111-120. doi: 10.1007/bf01731581

Kumar, S., Stecher, G., Li, M., Knyaz, C., and Tamura, K. (2018). MEGA X: molecular evolutionary genetics analysis across computing platforms. Mol. Biol. Evol. 35, 1547-1549. doi: 10.1093/molbev/msy096

Kurganov, E., Saito, S., Tanaka Saito, C., and Tominaga, M. (2017). Requirement of extracellular $\mathrm{Ca}^{2+}$ binding to specific amino acids for heat-evoked activation of TRPA1. J. Physiol. 595, 2451-2463. doi: 10.1113/jp274083

Kurganov, E., Zhou, Y., Saito, S., and Tominaga, M. (2014). Heat and AITC activate green anole TRPA1 in a membrane-delimited manner. Pflugers Arch. Eur. J. Physiol. 466, 1873-1884. doi: 10.1007/s00424-013-1420-z

Lailvaux, S. P., and Irschick, D. J. (2007). Effects of temperature and sex on jump performance and biomechanics in the lizard Anolis carolinensis. Funct. Ecol. 21, 534-543. doi: 10.1111/j.1365-2435.2007.01263.x

Li, T., Saito, C. T., Hikitsuchi, T., Inoguchi, Y., Mitsuishi, H., Saito, S., et al. (2019). Diverse sensitivities of TRPA1 from different mosquito species to thermal and chemical stimuli. Sci. Rep. 9:20200. doi: 10.1038/s41598-019-56639-w

Losos, J. B. (2008). Phylogenetic niche conservatism, phylogenetic signal and the relationship between phylogenetic relatedness and ecological similarity among species. Ecol. Lett. 11, 995-1003. doi: 10.1111/j.1461-0248.2008.01229.x

Lutterschmidt, W. I., and Hutchison, V. H. (1997). The critical thermal maximum: history and critique. Can. J. Zool. 75, 1561-1574. doi: 10.1139/z97-783

Myers, B. R., Sigal, Y. M., and Julius, D. (2009). Evolution of thermal response properties in a cold-activated TRP channel. PLoS One 4:e5741. doi: 10.1371/ journal.pone.0005741

Neely, G. G., Keene, A. C., Duchek, P., Chang, E. C., Wang, Q. P., Aksoy, Y. A., et al. (2011). TrpA1 regulates thermal nociception in Drosophila. PLoS One 6:e24343. doi: 10.1371/journal.pone.0024343

Oda, M., Ogino, H., Kubo, Y., and Saitoh, O. (2019). Functional properties of axolotl transient receptor potential ankyrin 1 revealed by the heterologous expression system. NeuroReport 30, 323-330. doi: 10.1097/wnr.0000000000001197

Orme, D., Freckleton, R., Thomas, G., Petzoldt, T., Fritz, S., Isaac, N., et al. (2018). The Caper Package: Comparative Analysis of Phylogenetics and Evolution in R. $R$ package, v. 1.0.1.

Oswald, M., Rymarczyk, B., Chatters, A., and Sweeney, S. T. (2011). A novel thermosensitive escape behavior in Drosophila larvae. Fly 5, 304-306. doi: 10.4161/fly.5.4.17810

Pagel, M. D. (1999). Inferring the historical patterns of biological evolution. Nature 401, 877-884. doi: 10.1038/44766

Paradis, E., Claude, J., and Strimmer, K. (2004). APE: analyses of phylogenetics and evolution in R language. Bioinformatics 20, 289-290. doi: 10.1093/ bioinformatics/btg412

Patapoutian, A., Peier, A. M., Story, G. M., and Viswanath, V. (2003). ThermoTRP channels and beyond: mechanisms of temperature sensation. Nat. Rev. Neurosci. 4, 529-539. doi: 10.1038/nrn1141

Peng, G., Shi, X., and Kadowaki, T. (2015). Evolution of TRP channels inferred by their classification in diverse animal species. Mol. Phylogenet. Evol. 84, 145-157. doi: 10.1016/j.ympev.2014.06.016

Prober, D. A., Zimmerman, S., Myers, B. R., McDermott, B. M., Kim, S. H., Caron, S., et al. (2008). Zebrafish TRPAl channels are required for chemosensation but not for thermosensation or mechanosensory hair cell function. J. Neurosci. 28, 10102-10110. doi: 10.1523/jneurosci.2740-08.2008

R Development Core Team (2018). R: a Language and Environment for Statistical Computing. Vienna: R Foundation for Statistical Computing.

Revell, L. J. (2010). Phylogenetic signal and linear regression on species data. Methods Ecol. Evol. 1, 319-329. doi: 10.1111/j.2041-210x. 2010.00044.x

Revell, L. J. (2012). Phytools: an R package for phylogenetic comparative biology (and other things). Methods Ecol. Evol. 3, 217-223. doi: 10.1111/j.2041-210x. 2011.00169.x

Rosenzweig, M., Brennan, K. M., Tayler, T. D., Phelps, P. O., Patapoutian, A., and Garrity, P. A. (2005). The Drosophila ortholog of vertebrate TRPA1 regulates thermotaxis. Genes Dev. 19, 419-424. doi: 10.1101/gad.1278205

Ruibal, R. (1961). Thermal relations of five species of tropical lizards. Evolution 15, 98-111. doi: 10.2307/2405846

Saito, S., and Tominaga, M. (2017). Evolutionary tuning of TRPA1 and TRPV1 thermal and chemical sensitivity in vertebrates. Temperature 4, 141-152. doi: 10.1080/23328940.2017.1315478

Saito, S., Banzawa, N., Fukuta, N., Saito, C. T., Takahashi, K., Imagawa, T., et al. (2014). Heat and noxious chemical sensor, chicken TRPA1, as a target of bird repellents and identification of its structural determinants by multispecies functional comparison. Mol. Biol. Evol. 31, 708-722. doi: 10.1093/molbev/ msu001

Saito, S., Hamanaka, G., Kawai, N., Furukawa, R., Gojobori, J., Tominaga, M., et al. (2017). Characterization of TRPA channels in the starfish Patiria pectinifera: involvement of thermally activated TRPA1 in thermotaxis in marine planktonic larvae. Sci. Rep. 7:2173. doi: 10.1038/s41598-017-02171-8

Saito, S., Nakatsuka, K., Takahashi, K., Fukuta, N., Imagawa, T., Ohta, T., et al. (2012). Analysis of transient receptor potential ankyrin 1 (TRPA1) in frogs and lizards illuminates both nociceptive heat and chemical sensitivities and coexpression with TRP vanilloid 1 (TRPV1) in ancestral vertebrates. J. Biol. Chem. 287, 30743-30754. doi: 10.1074/jbc.m112.362194

Saito, S., Ohkita, M., Saito, C. T., Takahashi, K., Tominaga, M., and Ohta, T. (2016). Evolution of heat sensors drove shifts in thermosensation between Xenopus species adapted to different thermal niches. J. Biol. Chem. 291, 11446-11459. doi: 10.1074/jbc.m115.702498

Saito, S., Saito, C. T., Nozawa, M., and Tominaga, M. (2019). Elucidating the functional evolution of heat sensors among Xenopus species adapted to different thermal niches by ancestral sequence reconstruction. Mol. Ecol. 28, 3561-3571. doi: $10.1111 / \mathrm{mec} .15170$

Sato, A., Sokabe, T., Kashio, M., Yasukochi, Y., Tominaga, M., and Shiomi, K. (2014). Embryonic thermosensitive TRPA1 determines transgenerational diapause phenotype of the silkworm, Bombyx mori. Proc. Natl. Acad. Sci. U.S.A. 111, E1249-E1255. doi: 10.1073/pnas.1322134111

Schettino, L. R., Losos, J. B., Hertz, P. E., Queiroz, K., De, Chamizo, A. R., et al. (2010). The anoles of soroa: aspects of their ecologiacl relationships. Breviora 520, 1-22. doi: 10.3099/0006-9698-520.1.1

Sherman, E., and Levitis, D. (2003). Heat hardening as a function of developmental stage in larval and juvenile Bufo americanus and Xenopus laevis. J. Therm. Biol. 28, 373-380. doi: 10.1016/s0306-4565(03)00014-7

Stecher, G., Tamura, K., and Kumar, S. (2020). Molecular evolutionary genetics analysis (MEGA) for macOS. Mol. Biol. Evol. 37, 1237-1239. doi: 10.1093/ molbev/msz312

Story, G. M., Peier, A. M., Reeve, A. J., Eid, S. R., Mosbacher, J., Hricik, T. R., et al. (2003). ANKTM1, a TRP-like channel expressed in nociceptive neurons, is activated by cold temperatures. Cell 112, 819-829. doi: 10.1016/s0092-8674(03) 00158-2

Tattersall, G. J., Sinclair, B. J., Withers, P. C., Fields, P. A., Seebacher, F., Cooper, C. E., et al. (2012). Coping with thermal challenges: physiological adaptations to environmental temperatures. Compr. Physiol. 2, 2151-2202. doi: 10.1002/ cphy.c110055

Tominaga, M., and Caterina, M. J. (2004). Thermosensation and pain. J. Neurobiol. 61, 3-12. doi: 10.1002/neu.20079

Venkatachalam, K., and Montell, C. (2007). TRP channels. Annu. Rev. Biochem. 76, 387-417. doi: 10.1146/annurev.biochem.75.103004.142819

Vitt, L. J., and Caldwell, J. P. (2008). Herpetology: an Introductory Biology of Amphibians and Reptiles. Cambridge, MA: Academic press. 
Voets, T., Talavera, K., Owsianik, G., and Nilius, B. (2005). Sensing with TRP channels. Nat. Chem. Biol. 1, 85-92. doi: 10.1038/nchembio0705-85

Vriens, J., Nilius, B., and Voets, T. (2014). Peripheral thermosensation in mammals. Nat. Rev. Neurosci. 15, 573-589. doi: 10.1038/nrn3784

Wang, G., Gordon, T. N., and Rainwater, S. (2008). Maximum voluntary temperature of insect larvae reveals differences in their thermal biology. J. Therm. Biol. 33, 380-384. doi: 10.1016/j.jtherbio.2008.06.002

Wilson, M. A., and Echternacht, A. C. (1990). A comparison of heat and cold tolerance of two morphs of Anolis carolinensis (Iguanidae) from Southern Florida. J. Herpetol. 24, 330-333. doi: 10.2307/1564411
Conflict of Interest: The author declares that the research was conducted in the absence of any commercial or financial relationships that could be construed as a potential conflict of interest.

Copyright $\odot 2021$ Akashi. This is an open-access article distributed under the terms of the Creative Commons Attribution License (CC BY). The use, distribution or reproduction in other forums is permitted, provided the original author(s) and the copyright owner(s) are credited and that the original publication in this journal is cited, in accordance with accepted academic practice. No use, distribution or reproduction is permitted which does not comply with these terms. 